\title{
THICK FILM CIRCUIT LAYOUT AND EXTRACTION OF PARAMETERS USING THE MAGIC LAYOUT EDITOR
}

\author{
J.N. AVARITSIOTIS \\ National Technical University of Athens, Department of Electrical Engineering, Division of Computer \\ Engineering, 157-73 Zographou, Athens-Greece
}

A Technology file for the Magic layout editor has been developed in order to enable thick film circuit designers to evolve fast solutions for layout with design rule checking, and plotting of the masks of multilayer hybrid circuits. The hybrid design system developed provides for device placement, and automatic routing techniques, in thick film circuits. Device placement is done by recalling from the library the pad pattern that corresponds to the device to be positioned. Automatic routing is implemented not only in multilayered circuits but also in single-layer technology (i.e. two possible types of metal routed on the bare substrate) where dielectric tiles are automatically created at metal crossings, when an electric contact is not desired.

\section{INTRODUCTION}

The Magic version 4 graphical layout editor included in the 1986 VLSI Tools release from the University of California at Berkeley ${ }^{1}$ is a popular package for graphics generation, circuit extraction, and simulation for nMOS and CMOS VLSI circuits. Magic is technology-independent and consequently requires a technology file to describe the layers and the design rules of a particular fabrication technology.

Also, Magic includes incremental design rule checking, automatic routing, CIF (Caltec Intermediate Form) and GDSII (CALMA format) input and output, allowing for interfacing with various plotting devices and a plotting feature which permits areas of the layout to be zoomed or compacted, without having to worry about design-rule violations being created. Magic is based on the Mead-Conway style of design. This means that it uses simplified design rules and circuit structures. The simplifications result in slightly less dense circuits since Magic uses a Manhattan layout style (i.e. edges are vertical or horizontal) and lambda scaled grid, where lambda is the size of the smallest feature which can be drawn. The Manhattan style limits feature shapes to those that follow the $\mathrm{X}$ and $\mathrm{Y}$ axes, and such a conservative design costs $5-10 \%$ in layout density. The physical dimension corresponding to a lambda unit is specified during circuit extraction or CIF or GDSII file generation.

In Magic, a circuit layout is a hierarchical collection of cells. Each cell contains: coloured shapes, that define the circuit's structure, and its eventual function; textual labels attached to the coloured shapes, in order to be able to manage the layout and provide a way of communicating information between various synthesis and analysis tools; and subcells which are instances of other cells. 
When a layout is edited with Magic, the system automatically checks design rules incrementally, meaning that the DRC acts on the most recently modified area in the layout and flags layout errors if there are any. In the case of finding a rule violation, Magic will display little white dots in the vicinity of the violation. The dots will remain on layout until the violation is corrected. Correction of the violation is facilitated with the use of special commands which allow the user to get information about the errors occurred.

In Magic, mask layers are represented by tiles, which are layout segments of maximum width and length in a contiguous region and are made of material of the same abstract layers. The design rules are applied at the edges between the tiles in the same plane, in a hierarchical approach; each time a structure is utilized, only the interaction between the structure and its surroundings is checked ${ }^{2}$. The algorithm analyzes the hierarchical nature of the design and checks the DRC violations by examining each cell and the interaction of the cells the first time they are encountered.

The 1986 release of VLSI Berkeley tools incorporated also, a circuit or node extractor program that takes a CIF file and produces as output the underlying electrical network, and other information such as the length/width ratio of each transistor, the resistance and capacitance of each connecting wire, etc. The output of the circuit extractor can only be used by switch-level simulators, such as ESIM ${ }^{3}$, to verify functional correctness, whereas the use of a parasitic extractor instead would provide data for timing simulators.

Magic runs on several hardware configurations; in our case Magic was installed on a SUN 3/60 work station.

\section{THE TECHNOLOGY FILE}

The technology file (see Appendix I) contains all information on what planes will contain the tiles, what layers will be used, and by what names and colours they will be represented. Also, all design rules, such as minimum widths, spacings, overlaps, and extensions are specified. Descriptions of what new tiles can be composed by painting one layer over another are specified in the technology file. Magic can be directed as to which technology is to be used by specifying a flag on the command line when Magic is run.

The version of the technology file proposed here is based on the technology file for scmos and it may consequently replace it without the need to recompile Magic. This means that any system running Magic with the standard technology file for scmos, can without any other modification run Magic with the technology file for hybrid circuits. The technology file for hybrid circuits supports one and two metallization layer technologies. The designer is allowed to choose from up to four resistor pastes per metallization layer. The scmos technology file with minor changes can also support three, four, etc. metallization layer technologies but recompilation of magic would be needed.

This implementation exploits fully Magic's built-in capabilities for circuit parameter extraction, automatic routing, and design rule checking. The circuit element 
parameters and the design rules may have to be altered to fit the technology that is going to be used for the implementation of the designed circuit. The design rules also have to be modified to conform with the requirements of each fabrication facility.

The two metallization layer technology is described in the following. The one metallization layer technology may be implemented by using only one of the two sets of resistor pastes and metal lines provided by the technology file. In the proposed implementation four planes have been used:

1st PLANE: The first plane includes the first metallization layer and the resistor pastes associated with it. There are also two types of pads: a metal-type for discrete components (i.e. metallization patterns for connecting to the circuit under design a] SMDs which must be soldered, and b] dies of ICs which must be wire bonded) and a solder-type for printing solder paste for subsequent reflow soldering of SMD's.

2nd PLANE: The second plane includes the second metallization layer and the associated resistor pastes and pads similar to the ones described for the first plane.

3rd PLANE: The third plane includes only the dielectric, and

4th PLANE: The fourth plane includes the overglazing only.

Furthermore, contacts have been defined between the two metallization planes. The pads of each metallization layer correspond to holes in the overglazing plane, and for that purpose they are defined as contacts between their home plane and the O-plane.

It is worth noting that the metallization planes are the most complex constructions. A metallization plane consists basically of the conductor pattern and the four resistor types of that plane. When a resistor paste overlaps a metallization line a new tile-type (a contact) is automatically generated according to the definitions introduced in the compose section. That action simplifies the work of the design rule checker in the area of metal-resistor overlaps, and it also facilitates the writing of reliable design rule checking sections. The other feature of the plane in question is the pad. As it has already been mentioned two main categories of pads have been provided. A wide range of pads of both categories have been included in the pad library, which has been added to the Magic files.

Another feature of the technology file developed is the possibility of contact with the second metallization plane. In order to keep the design as clean as possible the only tile types of the two metallization layers that are allowed to overlap are the two metallization lines. At the crossovers, where vias are not formed, a patch of dielectric is automatically generated in the CIF output file created by Magic, as shown at the bottom part of Fig. 1. The cif output section's part that generates the dielectric mask is the following:

\author{
layer DI $\mathrm{m} 1$ \\ and $\mathrm{m} 2$ \\ grow 300 \\ or di
}




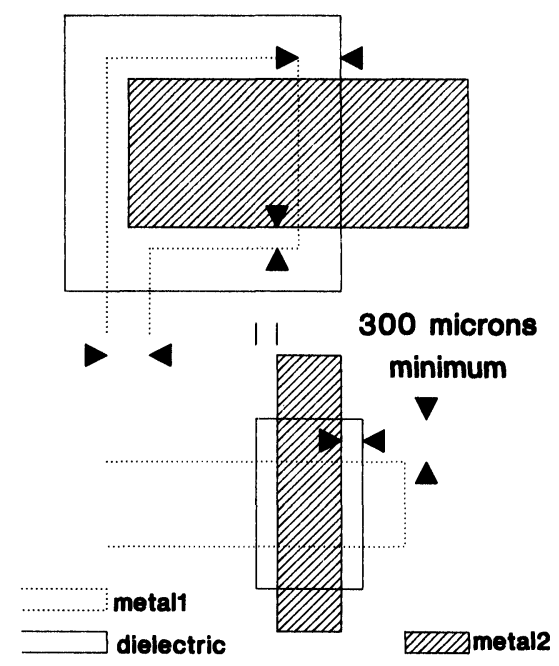

FIGURE 1 Design rules for capacitors and isolated metal crossings.

This enables the free use of the built-in automatic router, something which was not possible in a previous implementation ${ }^{4}$.

Moreover, specific thick film process rules and parameters ${ }^{5.6}$ have been inserted in the drc and extract sections.

It must be noted that special care has been taken in the selection of the colours for each tile type in such a way that what is seen on the graphics screen is always comprehensible.

It is apparent from the previous description that wirebonding sites, die pads, pads for SMDs, and resistor terminations may be placed by the designer either at

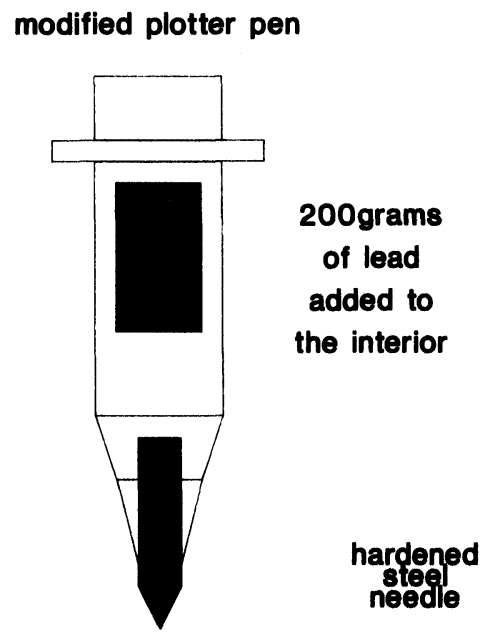

FIGURE 2 The schematic of the home made cutting tool. 
the 1st conductor's or the 2 nd conductor's plane. This gives a great flexibility to the designer: for example it allows the designer to use an inexpensive conductor such as palladium-silver on the lower level, where good wirebonding characteristics are not required; the upper level of metal may be screened with a gold paste, which is known to have excellent wirebonding characteristics and it may allow for eutectic die attachment.

Copies of our designs may be obtained on a X-Y plotter using $\mathrm{Vic}^{7}$, SOLO $1400^{8}$ or any other software packet that takes as an input a .CIF file and allows for the driving of a X-Y plotter. The same programs have been used to create the actual masks on rubylith, by replacing one of the pens with a home-made cutting tool, as only one layer at a time may be drawn on the X-Y plotter. A schematic picture of the cutting tool is shown in Fig. 2.

\section{DESIGN RULES}

Minimum widths are $300 \mu \mathrm{m}$ for metal lines, $1300 \mu \mathrm{m}$ for resistors, $500 \mu \mathrm{m}$ for die pads, wirebond sites, solder prints (to relow solder SMDs) and I/O pads, $1000 \mu \mathrm{m}$ for SMD-pads. Minimum spacings are $300 \mu \mathrm{m}$ between conductor and resistor patterns.

Also, minimum overlaps of $300 \mu \mathrm{m}$ have been implemented for printed resistors and SMDs to allow for misalignment, as shown in Fig. 3.
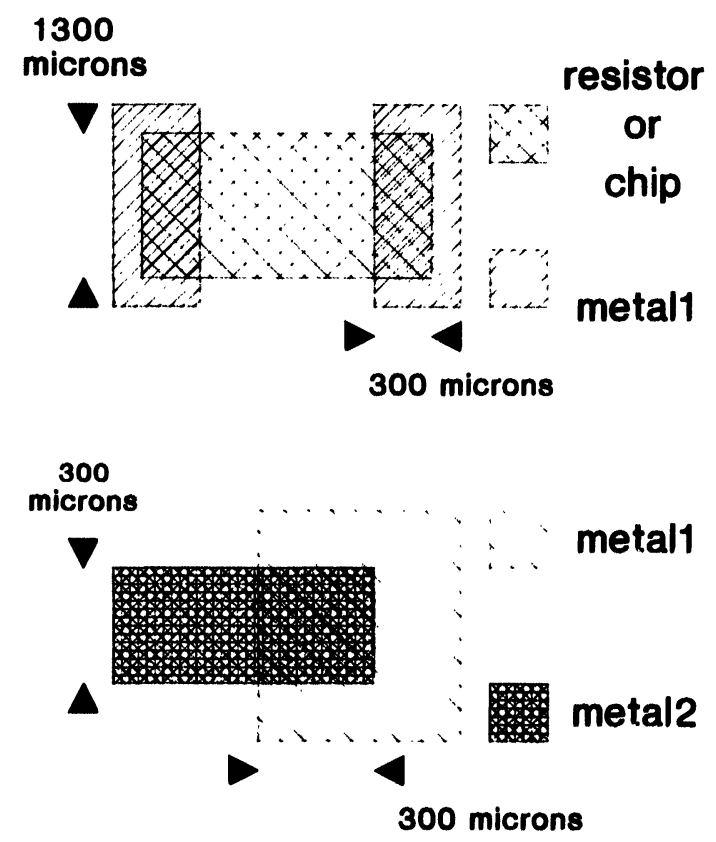

FIGURE 3 Overlap and extension design rules for discrete components, screen printed resistors, and metal lines. 


\section{THE EXTRACTOR}

Magic's extractor computes from the layout the information needed to run simulation tools such as Crystal. This information includes the connectivity, and the resistance and if required the parasitic capacitance of nodes.

Node resistances comprised entirely of a single type of material are calculated, in the case of simple rectangles, together with the perimeter and area of the resistor. The resistance, however, is always taken in the longer dimension of the rectangle. In the case of more complicated layouts, i.e. top hats, etc, the program computes the node's total perimeter and area and subsequently approximates the value of the resistor with that of a rectangle that has the same perimeter and area.

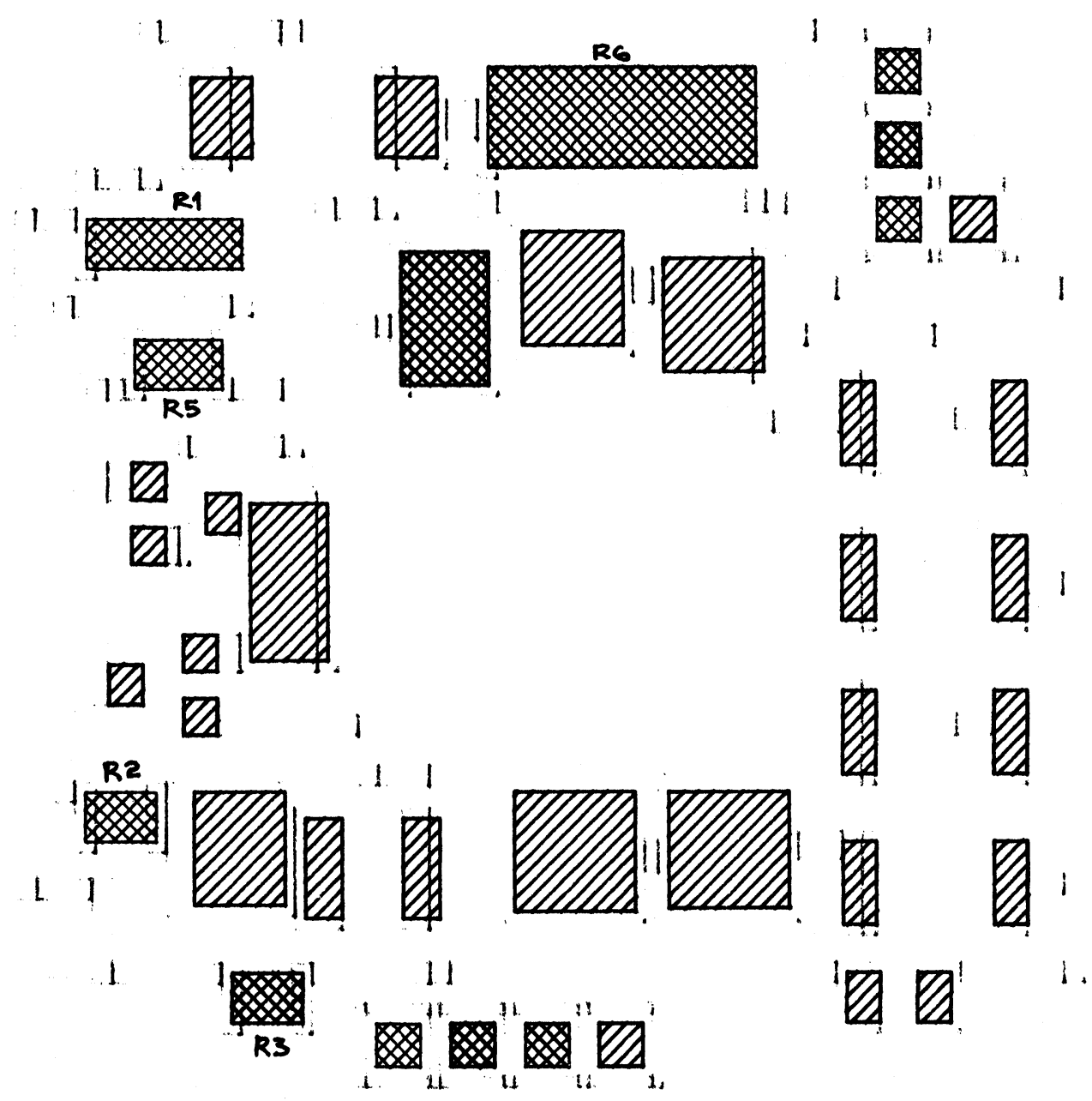

FIGURE 4 Actual pen plot of the electret-type microphone layout. 
Three types of internodal capacitance are extracted:

a] Overlap capacitance between two different types of conducting materials, separated by a dielectric, when they overlap,

b] Sidewall capacitance between the vertical edges of two pieces of the same type of conducting material, and

c] Sidewall overlap capacitance between the vertical edge of one type of conducting material and the horizontal surface of a second type of conducting material that overlaps the first.

The extracted values of parasitics of a thick film circuit which has been laid out with the Magic editor is considered to be valuable to the designer since it allows for a quick verification of the values of the resistors and capacitors designed. It also permits a good estimate of the minimum area of the ceramic substrate required for the implementation of the circuit.

\section{AN EXAMPLE}

As a demonstration an electret-type microphone circuit was designed and constructed using a palladium silver paste, four different resistor pastes, and SMDs for the capacitors and the diodes, using the hybrid design system developed. Fig. 4 shows the actual pen plot layout showing, in a 5:1 scale, the metal, the resistors and the solder pads. Since hierarchical designs are supported, the pad frames of the SMDs together with the solder paste pads, and also the alignment pattern containing substrate and layer-to-layer alignment marks can be saved in a library as separate objects to be called when needed. The remaining of the layout of the circuit was constructed by hand using the painting and wiring features of Magic. Since this design was done with conservative design rules, a quick $5 \mathrm{X}$ ruby-cutting was performed using our home-made cutting tool on a HP-7475A X-Y plotter with little loss of accuracy. It is worth noting that the speed of the tool should be defined as $1 \mathrm{~cm} / \mathrm{sec}$ in order to ensure a good, deep cutting.

The extractor has been used in order to confirm the validity of the values of the resistors designed during the layout procedure. The results are given in Table 1.

Three different resistor pastes have been used: $10 \Omega /$ sq., $1 \mathrm{~K} \Omega /$ sq., and $100 \mathrm{~K} \Omega /$ sq., for the fabrication of six different resistors.

TABLE 1

Extraction results of the circuit in Fig. 4.

\begin{tabular}{lrcr}
\hline node & $\begin{array}{l}\text { Value } \\
\text { (Ohms) }\end{array}$ & $\begin{array}{l}\text { Area } \\
\text { (Magic Units) }\end{array}$ & $\begin{array}{l}\text { Perimeter } \\
\text { (Magic Units) }\end{array}$ \\
\hline R4 & 200,000 & 800 & 120 \\
R3 & 100,000 & 225 & 60 \\
R6 & 25 & 2220 & 208 \\
R1 & 2,667 & 600 & 110 \\
R2 & 1,000 & 225 & 60 \\
R5 & 1,333 & 300 & 70 \\
\hline
\end{tabular}




\section{CONCLUSIONS}

It has been shown that technology files for the Magic layout editor together with the use of other modified programs may facilitate the layout, design rule checking, extraction of circuit parameters, and plotting or rubylith cutting of one and two metal multi-layered hybrid circuits.

\section{ACK.NOWLEDGMENT}

The author wishes to thank recently graduated Electrical Engineering students G. Stamoulis and S. Achtidas for coding and debugging much of the technology files.

\section{REFERENCES}

1. W.S. Scott, R.N. Mayo, Gordon Hamachi, and J.K. Ousterhout, "VLSI Tools: Still More Works by the Original Artists", Report No. UCB/CSD 86/272, Computer Science Division (EECS), University of California, Berkeley, California 94720, 1986.

2. T. Whitney, "A Hierarchical Design-Rule Checking Algorithm", LAMBDA, 1st quarter 1981, p. 40.

3. R. Bryan, “An Algorithm for MOS Logic Simulation”, LAMDA, Fall 1980, p. 29.

4. K.J. Scoles, "Thick-Film Circuit Layout Using the Magic Layout Editor", IEEE Trans. Comp., Hybrids, Manuf. Technol., vol. 11 (3), pp. 267-269, September 1988.

5. A.B. Glaser and G.E. Subak-Sharpe, "Integrated Circuit Engineering", Chapter 9, p. 376, AddisonWesley 1977.

6. P.J. Holmes and R.G. Loasby, "Handbook of Thick Film Technology", Chapter 8, p. 152, Electrochemical Publications Ltd., 1976.

7. Northwest Laboratory for Integrated Systems, "VLSI Design Tools Reference Manual", Release 3.1, February 15, 1987, University of Washington.

8. European Silicon Structures, "SOLO 1200 User's Manual", pp. 6-37, Command ARTVIEW, Software Release 2.2.2. August 1988.

\section{APPENDIX I: The Technology File}

tech
scmos
end
planes
metal1,m1
metal2,m2
dielectric,di
overglazing,o
end
types
m1
m2
m1
m1
m1




\section{APPENDIX I (Continued)}

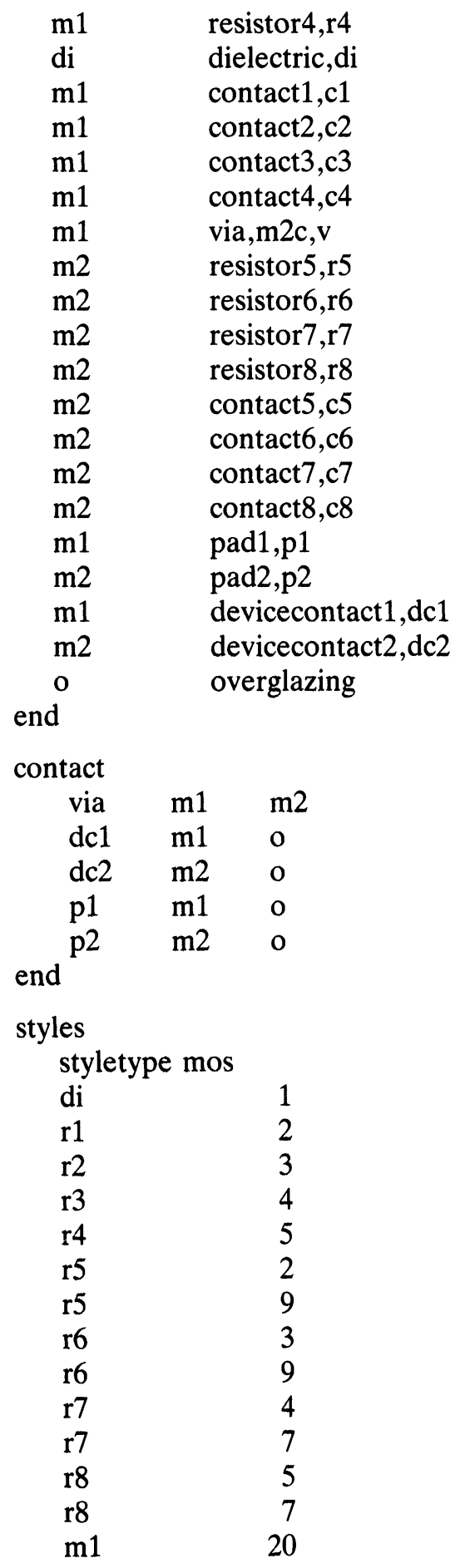




\section{APPENDIX I (Continued)}

$\begin{array}{lr}\mathrm{m} 2 & 21 \\ \mathrm{c} 1 & 2 \\ \mathrm{c} 1 & 20 \\ \mathrm{c} 1 & 32 \\ \mathrm{c} 2 & 3\end{array}$

c2 20

c2 32

c3 4

c3 20

c3 32

c4 5

c4 20

c4 32

c5 2

c5 9

c5 21

c5 32

c6 3

c6 $\quad 9$

c6 21

c6 32

$\begin{array}{ll}\text { c7 } & 4\end{array}$

$\begin{array}{ll}\text { c7 } & 7\end{array}$

c7 $\quad 21$

c7 $\quad 32$

c8 $\quad 5$

$\begin{array}{ll}c 8 & 7\end{array}$

c8 $\quad 21$

c8 $\quad 32$

via $\quad 20$

via 21

via 33

dc1 20

dc1 12

dc1 34

dc2 21

dc2 12

dc2 34

p1 20

p1 13

p1 33

p1 34

p2 21

p2 13




\section{APPENDIX I (Continued)}

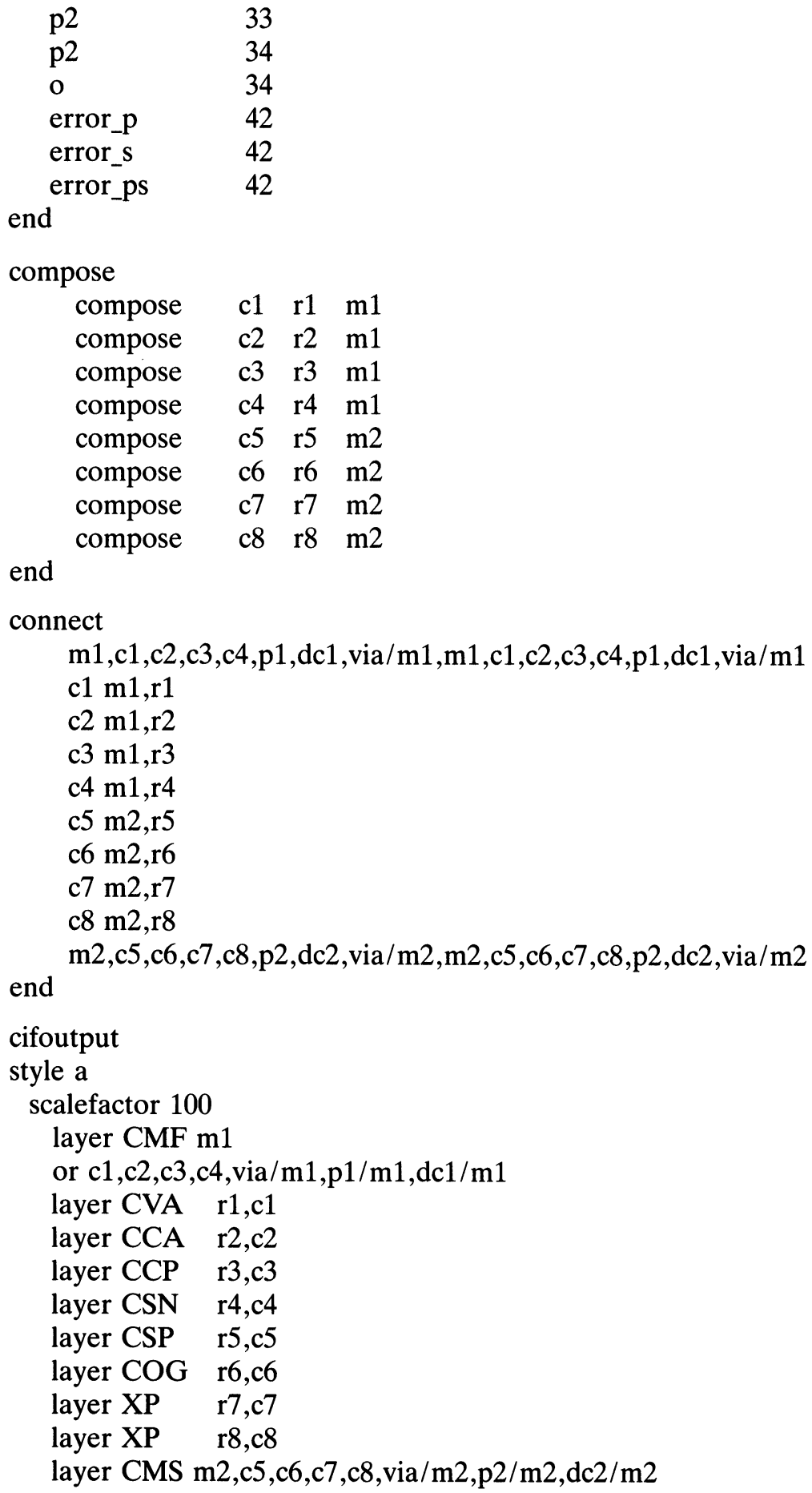




\section{APPENDIX I (Continued)}

layer CPG m1,r1,r2,r3,r4,c1,c2,c3,c4

and $\mathrm{m} 2, \mathrm{r} 5, \mathrm{r} 6, \mathrm{r} 7, \mathrm{r} 8, \mathrm{c} 5, \mathrm{c} 6, \mathrm{c} 7, \mathrm{c} 8$

grow 300

or $\mathrm{di}$

and-not via

layer CAA o

and-not $\mathrm{p} 1$

and-not $\mathrm{p} 2$

and-not $\mathrm{dc} 1$

and-not $\mathrm{dc} 2$

layer CWN dc1

or $\mathrm{dc} 2$

layer CWP p1

end

or $\mathrm{p} 2$

cifinput

style a

scalefactor 100

layer $\mathrm{m} 1 \mathrm{FML}$

labels FML

layer $\mathrm{r} 1 \mathrm{R} 1$

labels R1

layer $\mathrm{r} 2 \mathrm{R} 2$

labels R2

layer $\mathrm{r} 3 \mathrm{R} 3$

labels R3

layer $\mathrm{r} 4 \mathrm{R} 4$

labels R4

layer r5 R5

labels R5

layer $\mathrm{r} 6 \mathrm{R} 6$

labels R6

layer r7 R7

labels R7

layer $\mathrm{r} 8 \mathrm{R} 8$

labels R8

layer o OV

labels OV

layer p1 FML

and-not $\mathrm{OV}$

and $P D$

layer $\mathrm{p} 2 \mathrm{SML}$

and-not $\mathrm{OV}$

and PD 


\section{APPENDIX I (Continued)}

layer dc1 FML and-not $\mathrm{OV}$

and DV

layer dc2 SML and-not $\mathrm{OV}$ and DV

end

drc

edge4way via/m1 ( via,space) $/ \mathrm{m} 11 \sim(\mathrm{m} 2) / \mathrm{m} 2 \sim($ via $) / \mathrm{m} 11 \backslash$

"No dielectric near via" m2

width m1 3।

"Minimum conductor width must be 300 "

width $\mathrm{m} 23 \backslash$

"Minimum conductor width must be 300 "

width $\mathrm{r} 1, \mathrm{r} 2, \mathrm{r} 3, \mathrm{r} 413 \backslash$

"Minimum resistor width must be 1300 "

width r5,r6,r7,r8 13 ।

"Minimum resistor width must be 1300 "

width $\mathrm{c} 1, \mathrm{c} 2, \mathrm{c} 3, \mathrm{c} 431$

"Minimum resistor-conductor overlap must be $300^{\prime \prime}$ width $\mathrm{c5}, \mathrm{c6}, \mathrm{c7}, \mathrm{c8} 3$ ।

"Minimum resistor-conductor overlap must be $300^{\prime \prime}$

spacing $\mathrm{m} 1, \mathrm{via} / \mathrm{m} 1, \mathrm{c} 1, \mathrm{c} 2, \mathrm{c} 3, \mathrm{cc} 4, \mathrm{p} 1 / \mathrm{m} 1, \mathrm{dc} 1 / \mathrm{m} 1$

$\mathrm{m} 1, \mathrm{via} / \mathrm{m} 1, \mathrm{cc} 1, \mathrm{c} 2, \mathrm{c} 3, \mathrm{c} 4, \mathrm{p} 1 / \mathrm{m} 1, \mathrm{dc} 1 / \mathrm{m} 13$ touching_ok $\backslash$ "Minimum spacing must be $300^{\prime \prime}$ spacing $\mathrm{m} 2, \mathrm{via} / \mathrm{m} 2, \mathrm{c} 5, \mathrm{c} 6, \mathrm{c} 7, \mathrm{c} 8, \mathrm{p} 2 / \mathrm{m} 2, \mathrm{dc} 2 / \mathrm{m} 2 \mathrm{~m} 2, \mathrm{via} / \mathrm{m} 2, \mathrm{c} 5, \mathrm{c} 6, \mathrm{c} 7, \mathrm{c} 8, \mathrm{p} 2 /$ $\mathrm{m} 2, \mathrm{dc} 2 / \mathrm{m} 23$ touching_ok \"Minimum spacing must be 300 " spacing $\mathrm{r} 1 \mathrm{c} 1, \mathrm{r} 1,3$ touching_ok "Minimum spacing must be $300^{\prime \prime}$ spacing r2 c2,r2 3 touching_ok "Minimum spacing must be $300^{\prime \prime}$ spacing r3 c3,r3 3 touching_ok "Minimum spacing must be $300^{\prime \prime}$ spacing r4 c4,r4 3 touching_ok"Minimum spacing must be $300^{\prime \prime}$ spacing r5 c5,r5 3 touching_ok "Minimum spacing must be $300^{\prime \prime}$ spacing r6 c6,r6 3 touching_ok "Minimum spacing must be 300" spacing r7 c7,r7, 3 touching_ok "Minimum spacing must be $300^{\prime}$ spacing r8 c8,r8 3 touching_ok "Minimum spacing must be $300^{\prime \prime}$ edge4way r1 space $3 \mathrm{r} 1$,c1, space space 3 "Minimum spacing must be 300 " edge 4 way r2 space 3 r2,c2, space space 3 "Minimum spacing must be 300 " edge 4 way r3 space 3 r3,c3, space space 3 "Minimum spacing must be 300 " edge4way $\mathrm{r} 4$ space $3 \mathrm{r} 4$,c4, space space 3 "Minimum spacing must be 300 " edge4way r5 space 3 r5,c5,space space 3 "Minimum spacing must be $300^{\prime \prime}$ edge4way r6 space $3 \mathrm{r} 6, \mathrm{c} 6$,space space 3 "Minimum spacing must be 300 " edge 4 way r 7 space $3 \mathrm{r} 7, \mathrm{c} 7$, space space 3 "Minimum spacing must be $300^{\prime \prime}$ edge4way r8 space $3 \mathrm{r} 8$,c 8 ,space space 3 "Minimum spacing must be $300^{\prime}$ edge 4 way $\mathrm{c} 1, \mathrm{c} 2, \mathrm{c} 3, \mathrm{c} 4$ space $3 \mathrm{~m} 1$ space 3 "Minimum overlap must be $300^{\prime}$ edge4way c5,c6,c7,c8 space $3 \mathrm{~m} 2$ space 3

"Minimum overlap must be 300 " edge4way 


\section{APPENDIX I (Continued)}

$\mathrm{m} 2, \mathrm{c5}, \mathrm{c} 6, \mathrm{c} 7, \mathrm{cc} 8, \mathrm{r} 5, \mathrm{r} 6, \mathrm{r} 7, \mathrm{r} 8, \mathrm{dc} 2 / \mathrm{m} 2, \mathrm{p} 2 / \mathrm{m} 2, \mathrm{via} / \mathrm{m} 2$ space $3 \sim(\mathrm{dc} 1 / \mathrm{m} 1, \mathrm{p} 1 / \mathrm{m} 1) / \mathrm{m} 1$ space $3 \backslash$ "Metal2 layer types may not overlap metal1 pads" m1 edge4way p1/ $\mathrm{m} 1, \mathrm{dc} 1 / \mathrm{m} 1 \sim(\mathrm{p} 1 / \mathrm{m} 1, \mathrm{dc} 1 / \mathrm{m} 1) / \mathrm{m} 13 \sim(\mathrm{m} 2, \mathrm{c} 5, \mathrm{c} 6, \mathrm{c} 7, \mathrm{c} 8, \mathrm{r} 5, \mathrm{r} 6, \mathrm{r} 7, \mathrm{r} 8, \mathrm{dc} 2 / \mathrm{m} 2, \mathrm{p} 2 /$ $\mathrm{m} 2, \mathrm{via} / \mathrm{m} 2) / \mathrm{m} 2 \sim(\mathrm{p} 1 / \mathrm{m} 1, \mathrm{dc} 1 / \mathrm{m} 1) / \mathrm{m} 13 \backslash$ "Metal2 layer types may not overlap metal1 pads" m2 width 15 "Minimum width 500 " width p2 5 "Minimum width 500 " width dc1 10 "Minimum width 1000 " width dc2 10 "Minimum width 1000 " end

extract

style a

lambda 100

step 100

resist $\mathrm{m} 10$

resist $\mathrm{r} 10$

resist $\mathrm{c} 10$

end

wiring

contact via $4 \mathrm{~m} 10 \mathrm{~m} 20$

end

router

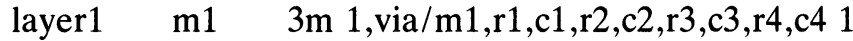

layer2 $\mathrm{m} 2 \quad 3 \mathrm{~m} 2, \mathrm{via} / \mathrm{m} 21$

contacts via 3

end

gridspacing 3

plowing

end

plot

end 

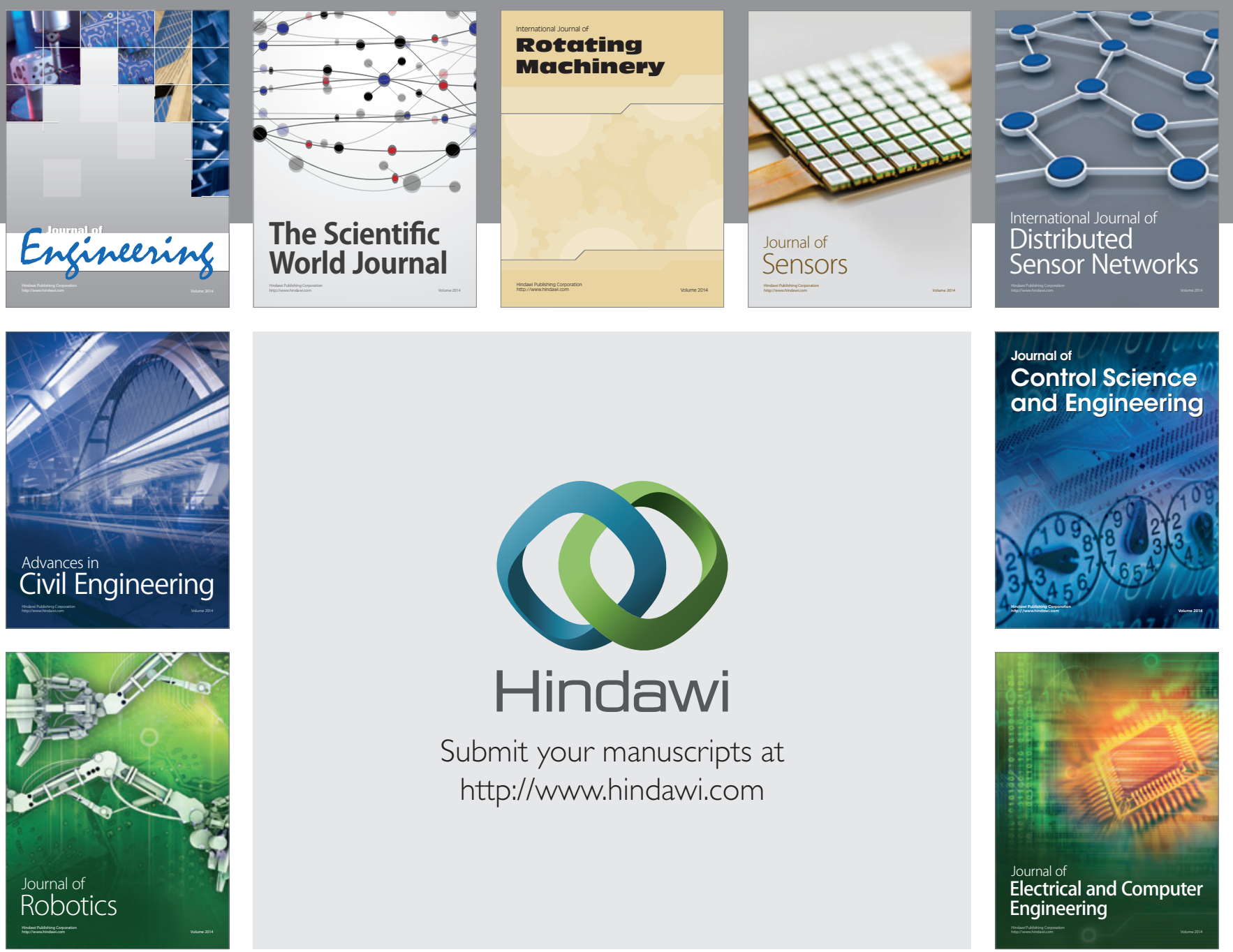

Submit your manuscripts at

http://www.hindawi.com
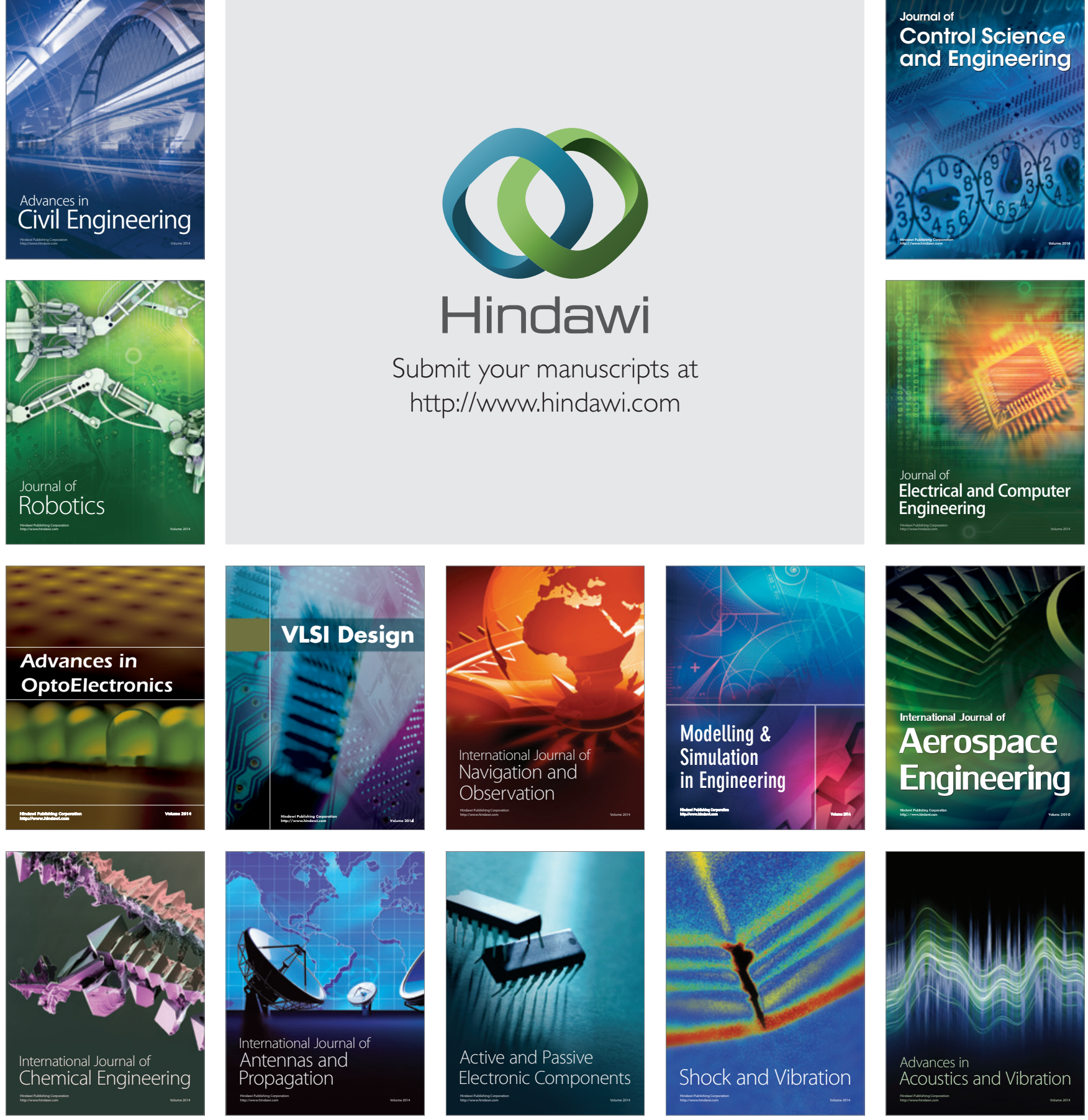